\title{
The role of Dickkopf-3 overexpression in esophageal adenocarcinoma
}

\author{
Zhuwen Wang, MS, ${ }^{\mathrm{a}}$ Lin Lin, MD, PhD, ${ }^{\mathrm{a}}$ Dafydd G. Thomas, MD, ${ }^{\mathrm{b}}$ Ernest Nadal, MD, \\ Andrew C. Chang, MD, ${ }^{a}$ David G. Beer, PhD, ${ }^{a}$ and Jules Lin, $\mathrm{MD}^{\mathrm{a}}$
}

\section{ABSTRACT}

Objectives: Ninety percent of patients with esophageal adenocarcinoma ultimately die of their disease, highlighting the need for novel therapeutic targets. The goal of this study was to define the functional significance of overexpression of Dickkopf-3 (DKK3) in esophageal adenocarcinoma.

Methods: $D K K 3$ expression was analyzed by real-time polymerase chain reaction in 95 chemonaive and 21 chemoresistant esophageal adenocarcinomas. The esophageal adenocarcinoma cell line OE33 was stably transfected with $D K K 3$ (OE33/DKK3) and evaluated using WST-1 (Roche, Basel, Switzerland), Matrigel (BD Biosciences, San Jose, Calif), endothelial tube formation, and chemosensitivity assays. Tumorigenesis was evaluated by injecting $1 \times 10^{6}$ OE33/DKK3 and vector cells in NOD/SCID $\gamma$ mice.

Results: $D K K 3$ was overexpressed (>2-fold) in 75.8\% (72/95) of esophageal adenocarcinomas. DKK3 protein was present at moderate to high levels in $46.8 \%$ (29/62) of esophageal adenocarcinomas on tissue microarray. Stable transfection of $D K K 3$ significantly increased proliferation $(P<.05)$ and Matrigel invasion $(P<.001)$. Levels of SMAD4, a key mediator of the transforming growth factor- 3 pathway, increased after activin treatment of OE33/DKK3, and siSMAD4 significantly decreased Matrigel invasion, suggesting that DKK3 acts through the transforming growth factor- $\beta$ pathway. OE33/DKK3 cells increased endothelial tube formation and were significantly more resistant to 5-fluorouracil and cisplatin, and $D K K 3$ expression was significantly higher in chemoresistant esophageal adenocarcinomas $(P<.005)$. In NOD/SCID $\gamma$ mice, OE33/DKK3 cells resulted in tumors at all sites $(8 / 8)$, whereas vector cells grew in only 1 of 8 sites. Nodal metastases were also significantly increased in patients with esophageal adenocarcinomas highly overexpressing $D K K 3,28$ of $32(88 \%)$ versus 42 of $63(68 \%)(P<.05)$.

Conclusions: These findings suggest that DKK3 may be important in mediating invasion in esophageal adenocarcinoma and could be a novel target in the treatment and prevention of metastatic disease. ( $\mathrm{J}$ Thorac Cardiovasc Surg $2015 ; 150: 377-85)$

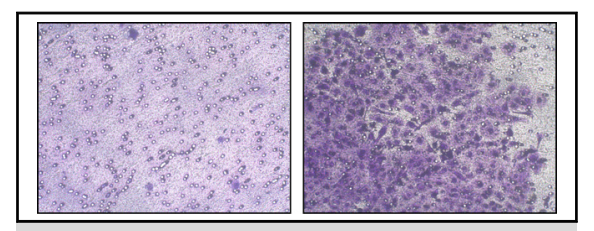

OE33/DKK3 had significantly increased Matrigel (BD Biosciences, San Jose, Calif) invasion compared with controls $(P<.001)$.

\section{Central Message}

DKK3 may mediate invasion in EAC and could be a novel target in the treatment and prevention of metastases.

\section{Perspective}

Most patients with EAC die of metastases. $D K K 3$ was significantly overexpressed in EAC and was associated with advanced tumors and nodal disease. Stable transfection of $D K K 3$ increases proliferation, invasion, and chemoresistance. DKK3 may be important in mediating invasion and could be a novel target in the treatment and prevention of metastatic disease.

See Editorial page 288.

\footnotetext{
From the ${ }^{\text {a Section }}$ of Thoracic Surgery, Department of Surgery and ${ }^{\mathrm{b}}$ Department of Pathology, University of Michigan Medical School, Ann Arbor, Mich.

Supported by the Thoracic Surgery Foundation for Research and Education Research Grant (JL), the Department of Surgery, University of Michigan Research Advisory Committee Grant (JL), and U54 CA163059 from the National Institutes of Health (DGB).

Received for publication Jan 13, 2015; revisions received March 31, 2015; accepted for publication May 2, 2015; available ahead of print June 18, 2015.

Address for reprints: Jules Lin, MD, Section of Thoracic Surgery, University of Michigan Medical Center, 1500 E. Medical Center Dr, 2120TC/5344, Ann Arbor, MI 48109-5344 (E-mail: juleslin@umich.edu).

$0022-5223 / \$ 36.00$

Copyright (c) 2015 by The American Association for Thoracic Surgery http://dx.doi.org/10.1016/j.jtcvs.2015.05.006
}

Supplemental material is available online.

Dickkopf-3 (DKK3) is a divergent member of the Wnt inhibitor family, ${ }^{1}$ and the significance of its interaction with the Wnt pathway is unclear. Although DKK1, 2, and 4 inhibit the Wnt pathway by binding to LRP5/6, DKK3 does not bind to these proteins. ${ }^{2}$ DKK3 binds to Kremen $1 / 2^{3}$; however, DKK3 is a secreted protein, and the significance of this intracellular interaction is uncertain. DKK3 was found to regulate fibroblast growth factor and Activin/Nodal through SMAD4, a central component of 


$$
\begin{aligned}
& \text { Abbreviations and Acronyms } \\
& \begin{aligned}
\text { BM } & =\text { Barrett's metaplasia } \\
\text { DKK3 } & =\text { Dickkopf-3 } \\
\text { EAC } & =\text { esophageal adenocarcinoma } \\
\text { FBS } & =\text { fetal bovine serum } \\
5-F U & =5 \text {-fluorouracil } \\
\text { PCR } & =\text { polymerase chain reaction } \\
\text { RT-PCR } & =\text { real-time polymerase chain reaction } \\
\text { SNP } & =\text { single nucleotide polymorphism } \\
\text { TGF } & =\text { transforming growth factor } \\
\text { VEGF } & =\text { vascular endothelial growth factor }
\end{aligned}
\end{aligned}
$$

the transforming growth factor (TGF) $\beta$ pathway, and stabilization of SMAD4 by DKK3 was found in the induction of mesoderm in Xenopus embryos. ${ }^{4}$

DKK3 has been proposed as a tumor suppressor, and overexpression of DKK3 suppresses cell growth and invasion of certain cancer cell lines. ${ }^{5}$ However, DKK3 is overexpressed in other cancers, including hepatocellular carcinoma and hepatoblastoma. ${ }^{6}$ DKK3 is a marker for neoangiogenesis in colon cancer, ${ }^{7}$ and microvessels expressing DKK3 were increased in glioma, non-Hodgkin's lymphoma, and melanoma. ${ }^{8}$ DKK3 has been associated with protection from apoptotic stress and with chemoresistance in Saos-2 osteosarcoma cells. ${ }^{9}$ By using Oncomine (www.oncomine.org), a web-based application that allows evaluation of gene expression using cancer profiling data including 25 esophageal datasets with 751 samples, there was significant overexpression of $D K K 3$ in esophageal adenocarcinomas (EACs) relative to Barrett's metaplasia (BM) and normal esophagus $(10.9$-fold; $P<.0001){ }^{10,11}$ Conversely, $D K K 3$ expression was significantly decreased in lung adenocarcinoma. The expression and function of DKK3 seem to be tissue and tumor specific.

The incidence of EAC has increased greatly, whereas the 5-year survival remains only $19 \%{ }^{12}$ Metastatic disease accounts for the majority of deaths from EAC. Although esophagectomy remains the primary treatment, there is an urgent need for novel therapies. In evaluating molecular changes in the progression from BM to EAC, overexpression of $D K K 3$ was identified in a significant subset of tumors. We found that a number of genes mediated by the $\operatorname{TGF} \beta$ pathway also were overexpressed, suggesting that this pathway is important in EAC. This study was undertaken to delineate the expression and role of DKK3 in EAC. We hypothesized that DKK3 is a mediator of the $\operatorname{TGF} \beta$ pathway in EAC and plays an important role in the proliferation and invasion of EAC. Inhibition of DKK3 and its downstream mediators could have a significant clinical impact on the treatment and prevention of micrometastatic disease, especially in patients with locally advanced or regional nodal disease.

\section{MATERIALS AND METHODS \\ Patients and Tissues}

This study was approved by the institutional review board, and after obtaining informed consent, tissues were obtained from patients undergoing esophagectomy at the University of Michigan. Specimens were transported in Dulbecco's Modified Eagle Medium (Invitrogen, Carlsbad, Calif) on ice and stored at $-80^{\circ} \mathrm{C}$. Samples with minimum $70 \%$ cellularity were identified using frozen sections, including 95 chemonaive and 21 chemoresistant EACs.

\section{Cell Lines}

Flo, OE19, and OE33 (Sigma-Aldrich, St Louis, Mo) were derived from EAC. Flo was grown in Dulbecco's Modified Eagle Medium (Invitrogen), and OE33 and OE19 were grown in Roswell Park Memorial Institute 1640 with $10 \%$ fetal bovine serum (FBS) (Atlanta Biologicals Inc, Flowery Branch, Ga) and $1 \%$ Antibiotic-Antimycotic (Invitrogen) at $37^{\circ} \mathrm{C}$ in $5 \%$ $\mathrm{CO}_{2} / 95 \%$ air. All cell lines and stable subclones underwent genotyping by the University of Michigan Sequencing Core to ensure cell line authenticity. To evaluate for chemosensitivity, cell lines were treated with cisplatin $(5 \mu \mathrm{g} / \mathrm{mL})$ and 5-fluorouracil $(5-\mathrm{FU})(10 \mu \mathrm{g} / \mathrm{mL})$ for 48 hours. Viability was assessed by WST-1 (Roche, Basel, Switzerland) and repeated in triplicate.

\section{Quantitative Reverse Transcription-Polymerase Chain Reaction}

Real-time polymerase chain reaction (RT-PCR) was performed using 20 ng of total RNA and $0.2 \mu \mathrm{mol} / \mathrm{L}$ of the forward and reverse primers. Cycling parameters included a $50^{\circ} \mathrm{C}$ hold for 2 minutes, $95^{\circ} \mathrm{C}$ hold for 10 minutes, 40 cycles at $95^{\circ} \mathrm{C}$ for 10 seconds, annealing for 15 seconds, and $72^{\circ} \mathrm{C}$ for 20 seconds. Significant differences in relative quantification were determined using the $2^{(-\Delta \Delta \mathrm{Ct})}$ method. Expression was normalized to GAPDH or $\beta$-actin. DKK3 primers were forward $5^{\prime}$-TGAGGAACTGATG GAGGACA- $3^{\prime}$ and reverse $5^{\prime}$-TTGCCAGGTTCACTTCTGAT- $3^{\prime}$.

\section{Western Blot}

Western blot was performed using a 1:1000 dilution of DKK3 antibody (Santa Cruz Biotechnology Inc, Dallas, Tex) and a 1:5000 dilution of goat anti-rabbit secondary antibody (Vector Laboratories, Burlingame, Calif). Although the calculated molecular weight of DKK3 is $38 \mathrm{kDa}$, the size on Western blot has been reported as 50 to $55 \mathrm{kDa}$ in reducing conditions due to glycosylation. ${ }^{13}$ For SMAD4, a 1:2000 dilution of SMAD4 antibody (Abcam, Cambridge, UK) was used with a 1:8000 dilution of anti-rabbit secondary antibody (Vector Laboratories). B-actin was used as a loading control with a 1:10000 dilution of B-actin antibody (Abcam) and a 1:10000 dilution of anti-mouse secondary antibody (Vector Laboratories).

\section{Tissue Microarray}

Tissue microarrays were constructed with formalin-fixed, paraffinembedded tissues from 73 patients, including 64 EAC, 8 dysplastic Barrett's mucosa, $11 \mathrm{BM}$, and 2 normal esophageal samples. ${ }^{14}$ Immunohistochemical staining was done on the DAKO Autostainer using DAKO (Carpinteria, Calif) LSAB + and 3,3'-diaminobenzidine as the chromogen. Dewaxed and rehydrated sections were labeled with DKK3 antibody (1:200 dilution; Santa Cruz Biotechnology Inc). Microwave citric acid epitope retrieval was performed for 20 minutes. Slides were counterstained with hematoxylin. Samples were scored using a scale of 0 (no staining), $1+(<10 \%$ staining), $2+(10 \%-50 \%)$ staining, or $3+$ $(\geq 50 \%) .{ }^{15}$ The scoring was repeated to ensure reproducibility.

\section{Construction of DKK3 Stable Cell Line}

A DKK3 mammalian expression construct (Plasmid 15496; Addgene, Cambridge, Mass) was polymerase chain reaction (PCR) amplified using 
primers containing EcoRI and XbaI restriction sites for directional cloning into the pcDNA3(+) Vector (V79020; Invitrogen). The insert was sequenced to confirm that it contained DKK3. DKK3 or empty vector constructs were transfected into OE33 or Flo using FuGENE 6 (Promega Corp, Madison, Wis). Selected clones were maintained in medium containing $200 \mu \mathrm{g} / \mathrm{mL}$ geneticin.

\section{Matrigel Invasion Assay}

$1 \times 10^{5}$ cells in serum-free Roswell Park Memorial Institute 1640 medium were seeded in the upper chamber of the 24-well invasion chamber system (BD Biosciences, San Jose, Calif), and 20\% FBS was added to the lower chamber as a chemoattractant. After 48 hours, noninvading cells and Matrigel (BD Biosciences) were removed with a cotton swab. Invasive cells on the lower side of the chamber were stained with crystal violet. Four $100 \times$ fields were counted, and assays were performed in duplicate.

\section{Single Nucleotide Polymorphism Array Analysis}

By using the Genome-Wide Human Sty I 250K single nucleotide polymorphism (SNP) Array (Affymetrix, Santa Clara, Calif), 73 EAC DNAs were genotyped. ${ }^{16}$ DKK3 copy number analysis was performed using a $\log 2$ copy number ratio exceeding 0.848 for amplifications and -0.737 for deletions. Genomic positions were mapped in the hg 18 genome build. SNP data were visualized using Integrative Genomics Viewer 1.3.1 (www.broadinstitute.org/igv).

\section{Endothelial Tube Formation}

Human microvascular endothelial cells (Cascade Biologics, Eugene, Ore) were stably transduced with lentiviral-expressed red fluorescent protein. OE33/DKK3 and OE33/Vector cells were stably transduced with lentiviral-expressed green fluorescent protein. Red fluorescent protein and green fluorescent protein-positive cells were sorted by flow cytometry. Selected human microvascular endothelial cells were cultured as monolayers on Attachment Factor coated plates with Medium 131 mixed with Microvascular Growth Supplement (Cascade Biologics) and 1\% FBS. ${ }^{17}$ Cells were applied to collagen gel polymerized 6-well plates $\left(2 \times 10^{5}\right.$ cells/well) and incubated for 16 hours. A second collagen gel layer was overlaid with $2 \times 10^{5} \mathrm{OE} 33 / \mathrm{DKK} 3$ or vector cells $/ \mathrm{mL}$. Vascular endothelial growth factor (VEGF) $(1 \mu \mathrm{g} / \mathrm{mL}$; R\&D Systems Inc, Minneapolis, Minn) was added to OE33/Vector with endothelial cells or endothelial cells alone as positive controls. Cells were incubated at $37^{\circ} \mathrm{C}$ for 7 days, and media was changed every 3 days. Experiments were performed in triplicate.

\section{Xenograft Models}

NOD/SCID $\gamma$ (NSG) mice were received from the Unit for Laboratory Animal Medicine at 6 to 8 weeks of age. All procedures were approved by the University Committee on Use and Care of Animals, and all animals received humane care in compliance with the Guide for the Care and Use of Laboratory Animals (www.nap.edu/catalog.php?record_id=12910). To evaluate whether stable transfection of $D K K 3$ increases the tumorigenicity of OE33, the number of cells injected was titrated to the lowest number resulting in a palpable tumor after flank injection of OE33/DKK3 but not OE33/Vector. Cells were passed through a $40-\mu \mathrm{m}$ filter. A single cell suspension was prepared with $100 \mu \mathrm{L}$ of saline and $50 \%$ Matrigel (BD Biosciences). $2 \times 10^{6}, 1 \times 10^{6}, 1 \times 10^{5}$, and $5 \times 10^{4}$ cells were injected into the flank of NSG mice. ${ }^{18}$ At $1 \times 10^{6}$ cells, OE33/DKK3 resulted in tumors at 5 weeks, whereas OE33/Vector cells did not. Each experimental group consisted of 4 mice injected with $1 \times 10^{6}$ cells in each flank for a total of 8 sites per cell line. Mice were sacrificed after 5 weeks.

\section{Oligonucleotide Microarray}

Total RNA was isolated from stably transfected OE33/DKK3 and OE33/Vector cells using QIAzol (Qiagen Inc, Valencia, Calif) and purified with miRNeasy spin columns (Qiagen Inc). RNA quality was confirmed by $1 \%$ agarose gel electrophoresis and A260:280 by NanoDrop 2000 spectrophotometer ratios. RNA quality was reassessed with the Agilent Bioanalyzer (Agilent Technologies, Santa Clara, Calif) after double-stranded cDNA and cRNA synthesis. Hybridization and normalization of the Human Gene 2.1 ST Gene Chip data (Affymetrix) were performed by the University of Michigan Cancer Center Microarray Core. A summary statistic was calculated for the 11 probe pairs for each gene with the robust multichip average method ${ }^{14}$ using the Affymetrix library of Bioconductor (www.bioconductor.org). Expression values for OE33/DKK3 were compared with OE33/Vector. Pathway analysis was performed using DAVID Bioinformatics Resources 6.7 (http://david.abcc.ncifcrf.gov). Results were confirmed using RT-PCR.

\section{Statistical Analysis}

Statistical analysis was performed using SPSS version 22 (SPSS Inc, Chicago, Ill). Categorical variables were analyzed using the Fisher exact or chi-square tests, and continuous variables were analyzed using the Student $t$ test. Survival was determined using the Kaplan-Meier method. For the analysis of the clinical characteristics, high DKK3 expression was defined as expression relative to Barrett's mucosa with a threshold greater than 2 standard deviations above the mean.

\section{RESULTS}

\section{DKK3 Is Overexpressed in Esophageal}

\section{Adenocarcinoma}

$D K K 3$ was overexpressed more than 2-fold in $75.8 \%$ (72/95) of EACs relative to BM using RT-PCR (Figure E1, A). Quantitative RT-PCR analysis of an additional 40 esophageal samples (6 BM, 7 BM/lowgrade dysplasia, 6 low-grade dysplasia, 7 high-grade dysplasia, and 14 EACs) showed significant overexpression in EAC in the progression from BM $(P<.05)$ (Figure E1, $B)$. The purpose was to identify molecular changes in the progression from BM to adenocarcinoma. Barrett's metaplasia was used as the control to avoid identifying genes with similar expression in BM and EAC but differential expression in normal esophagus. BM has been used as a reference control by other investigators. ${ }^{19}$ Significant overexpression of $D K K 3$ in 95 primary EACs was also confirmed relative to normal esophagus using RT-PCR.

Staining of DKK3 on tissue microarray (Table E1) showed moderate to high DKK3 expression (2-3+) in $46.8 \%(29 / 62)$ of EAC samples (Figure 1). Some $16.7 \%$ (2/12) of dysplastic samples and $20 \%(2 / 10)$ of BM samples had $2+$ staining, but unlike EAC, none had 3+ staining. Normal esophageal samples showed mild $1+$ DKK3 staining $(2 / 2)$.

To evaluate the mechanism of overexpression, SNP array analysis of 73 EACs was performed, and DKK3 was not amplified. Although treatment of OE33 with 5-azacytidine did not increase DKK3 expression, a combination of 5-azacytidine and trichostatin A increased DKK3 expression on Western blot, suggesting that histone acetylation is involved in the overexpression of $D K K 3$. 


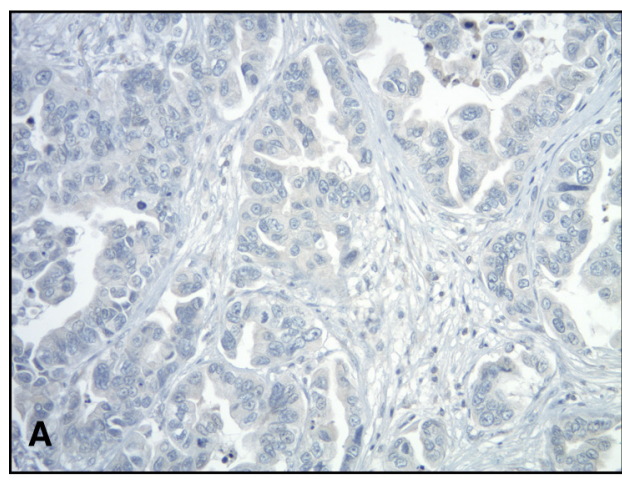

DKK3

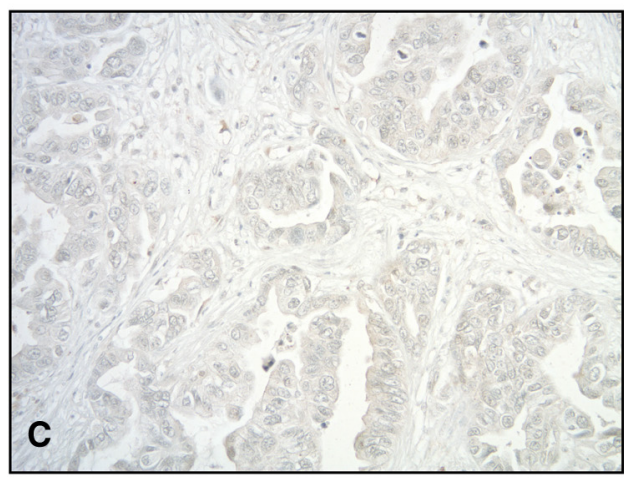

SMAD4

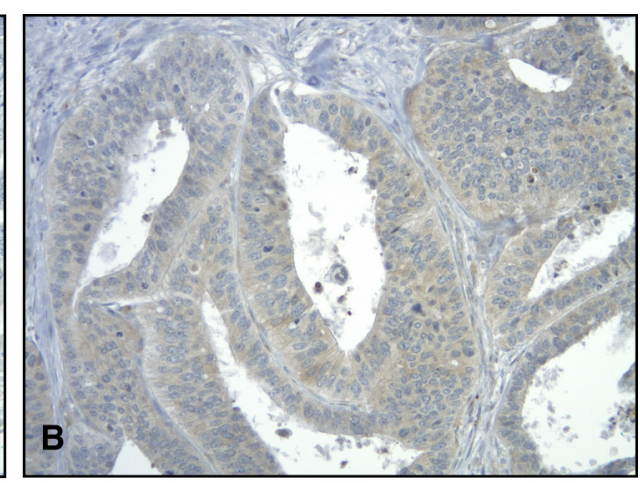

DKK3

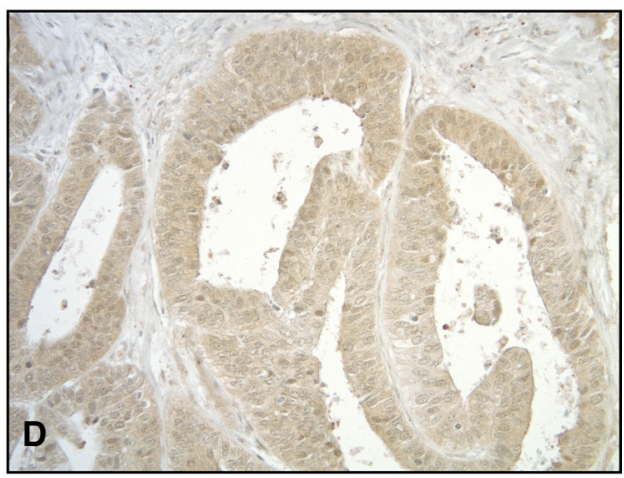

SMAD4

FIGURE 1. Representative sections from a tissue microarray showing low (A) and high (B) cytoplasmic DKK3 staining in 2 EACs. Moderate to high expression (2-3+) was found in 46.8\% (29/62) of EACs. Low (C) and high (D) SMAD4 staining correlated with DKK3 in the same tumors. Original magnifications are all $100 \times$. DKK3, Dickkopf-3.

\section{OE33 Transfection With DKK3 Increases Cell Proliferation and Matrigel Invasion}

DKK3 overexpression was confirmed on quantitative RT-PCR compared with native and vector controls and on Western blot, which also confirmed expression of a Flag tag. OE33, which does not natively express $D K K 3$, had significantly increased proliferation on WST-1 assay after DKK3 transfection compared with vector controls at 96 and 120 hours $(P<.005)$. OE33/DKK3 cells also had significantly greater Matrigel invasion $(P<.001)$ (Figure 2). To ensure that these results were not cell line specific, the EAC cell line Flo was transfected with DKK3 and confirmed increased proliferation and Matrigel invasion compared with vector controls.

\section{Transfection of DKK3 Leads to Stabilization of SMAD4 and Activation of the Transforming Growth Factor- $\beta$ Pathway}

To determine the mechanism of action of DKK3 in EAC, the TOP-flash TCF-reporter assay (Promega Corp., Madison, Wisc) was performed and showed no significant decrease in canonical Wnt pathway activation after transfection of $D K K 3$, a divergent member of the Wnt inhibitor family, into OE33 compared with native and vector controls. However, treatment of OE33/DKK3 cells with the TGF $\beta$ ligand activin increased SMAD4 protein on Western blot (Figure 3,A), suggesting its mechanism of action is through the $\operatorname{TGF} \beta$ pathway. Activin treatment also significantly increased proliferation in OE33/DKK3 compared with vector controls. Inhibition with siSMAD4 significantly decreased Matrigel invasion of OE33/DKK3 $(P<.001)$ (Figure $3, B$ and $C)$. In addition, low and high SMAD4 staining correlated with DKK3 expression in the same tumors on immunohistochemistry (Figure 1). DAVID pathway analysis of Human Gene ST 2.1 array expression induced by the transfection of $D K K 3$ in OE33 showed that the TGF $\beta$ pathway was significantly increased compared with OE33 vector control $(P<.05)$.

\section{DKK3 Overexpression Leads to Increased Neoangiogenesis}

Endothelial tube formation assay demonstrated increased angiogenesis in the presence of OE33/DKK3 compared with OE33 native, vector, and endothelial cell controls (Figure 4). Endothelial tube formation was similar to that 


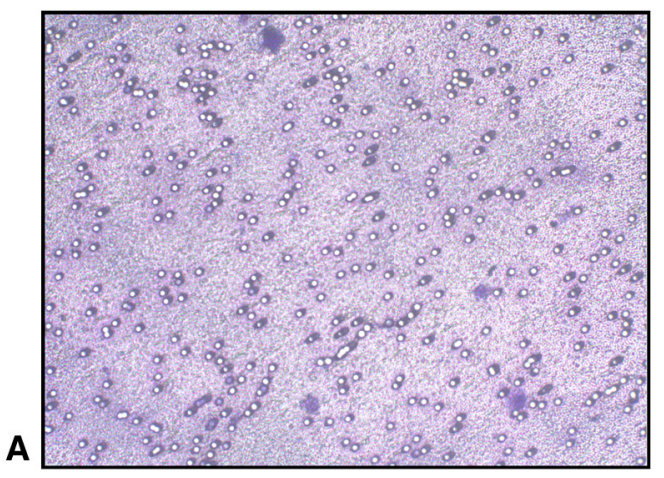

Native OE33

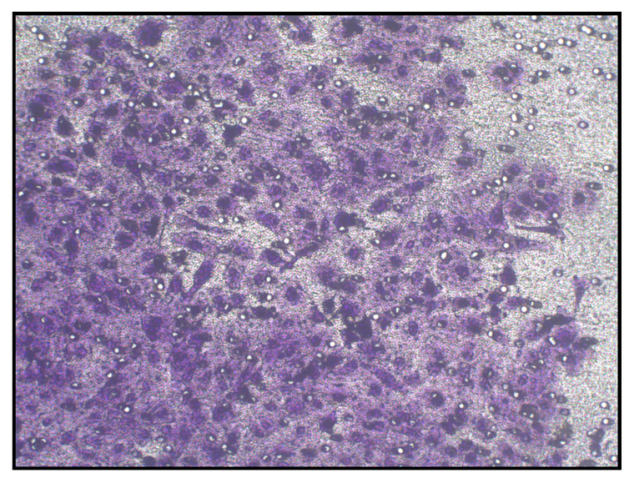

OE33/DKK3

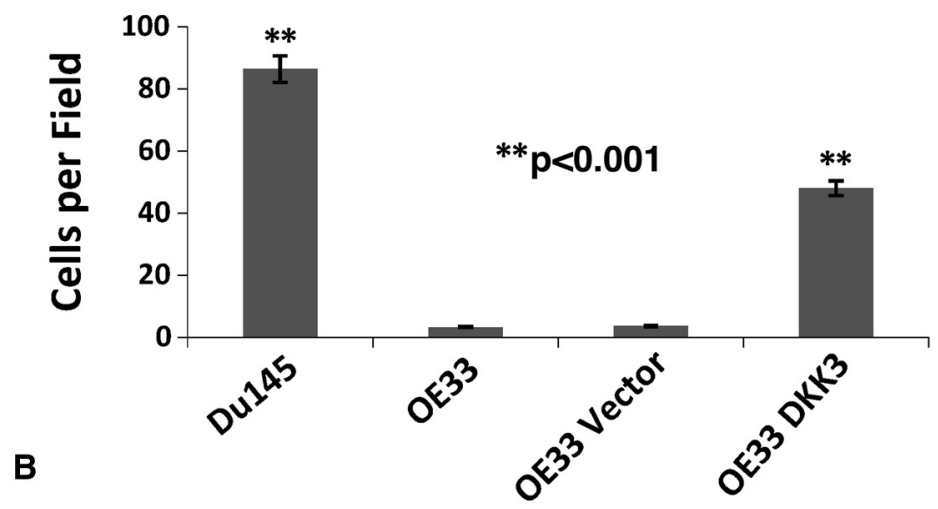

FIGURE 2. A, OE33/DKK3 had significantly increased Matrigel (BD Biosciences, San Jose, Calif) invasion compared with controls (**P<.001). Du145 prostate cancer cells served as a positive control. B, All assays were performed in duplicate. DKK3, Dickkopf-3.

seen after adding VEGF to the OE33/Vector and endothelial only positive controls.

\section{DKK3 Overexpression Increases Chemoresistance in Esophageal Adenocarcinoma Cells}

OE33/DKK3 cells were significantly more chemoresistant to 5-FU and cisplatin compared with vehicle control $(P<.05)$ (Figure 5, A). DKK3 expression was significantly higher on RT-PCR relative to BM in 21 chemoresistant EACs compared with 96 chemonaive EACs $(P<.005)$ (Figure $5, B$ ). To evaluate whether $D K K 3$ expression is induced by chemotherapy, OE33 and Flo were treated with cisplatin and 5-FU with no significant increase in $D K K 3$ expression.

Injection of OE33/DKK3 Cells in NOD/SCID $\gamma$ Mice Significantly Increases Incidence of Tumor Growth

NSG mice were injected in the flank with $1 \times 10^{6}$ cells, the lowest number of cells to produce tumors with OE33/ DKK3 but not OE33/Vector, as described in the "Materials and Methods" section. Five weeks after injection, only 1 of 8 OE33/Vector sites produced a palpable tumor $(2 \mathrm{~mm})$, whereas all OE33/DKK3 (8/8) tumor sites resulted in tumors $(4-5 \mathrm{~mm})$ (Figure E2). Tumors were confirmed pathologically to be consistent with EAC. DKK3 overexpression was also confirmed by RT-PCR and Western blot with no expression in the OE33/Vector tumor.

\section{Nodal Metastases Were Significantly Increased in Patients With Esophageal Adenocarcinomas Highly Overexpressing DKK3}

Clinical characteristics for 94 patients with chemonaive EACs, obtained before the routine use of preoperative chemotherapy, were analyzed for an association with $D K K 3$ overexpression (Table E2). Nodal metastases were significantly associated with $D K K 3$ overexpression, with 28 of $32(88 \%)$ with nodal disease compared with 42 of $62(68 \%)$ with lower DKK3 expression $(P=.047)$. The pathologic stage also was significantly higher with EACs highly overexpressing $D K K 3$, with 26 of $32(81 \%)$ EACs with stage III + IVa versus 36 of $62(58 \%)$ with lower $D K K 3$ expression $(P=.038)$. However, $D K K 3$ overexpression was not significantly associated with overall survival.

\section{DISCUSSION}

The TGF $\beta$ pathway is involved in proliferation, differentiation, and epithelial-mesenchymal transition, 


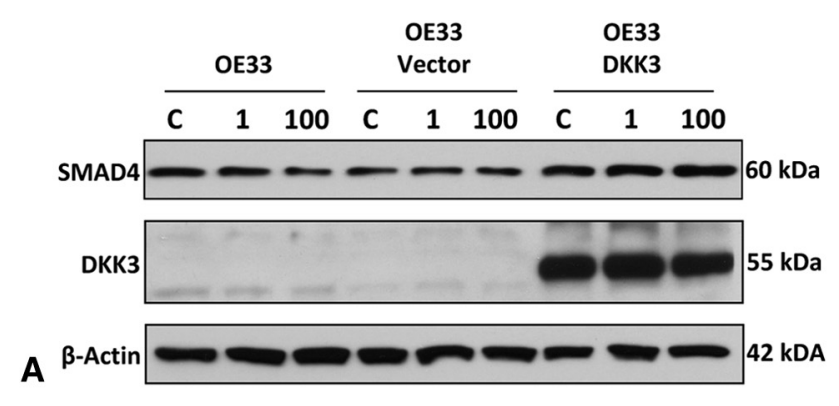

B

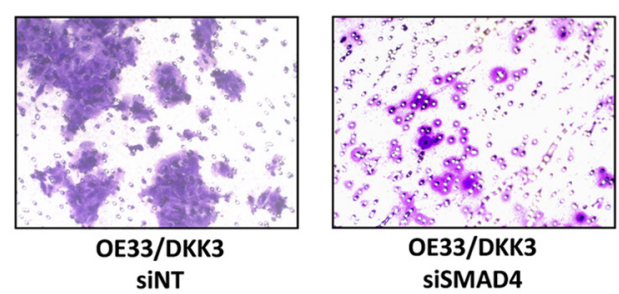

C

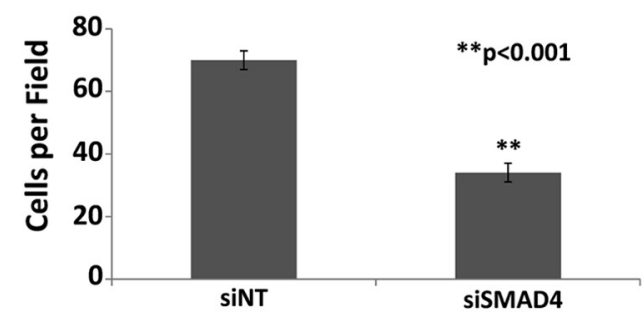

FIGURE 3. A. SMAD4 was significantly increased on Western blot in OE33/DKK3 after treatment with 1 or $100 \mathrm{ng} / \mathrm{mL}$ of activin compared with native and vector controls (C, carrier control). B and C, Treatment of OE33/DKK3 cells with siSMAD4 resulted in significantly less Matrigel invasion compared with a nontargeting control $(* * P<.001)$. DKK3, Dickkopf-3.

which has been associated with chemoresistance and tumor invasion. Although TGF $\beta$ is a tumor suppressor at early stages of carcinogenesis, advanced cancers are resistant to its growth inhibition and TGF $\beta$ promotes metastasis and invasion. ${ }^{20-22}$ TGF $\beta$ is overexpressed in EAC and related to a poor prognosis. ${ }^{23}$ DKK3 mediates the effects of activin, a TGF $\beta$ ligand, in Xenopus. ${ }^{4}$ Our results suggest that DKK3 acts independently of the canonical Wnt pathway in EAC and that the mechanism of action is mediated through the TGF $\beta$ pathway with DKK3 stabilizing SMAD4 protein. Transfection of DKK3 in OE33 resulted in increased activation of the TGF $\beta$ pathway. Various tumor biological processes were evaluated focusing on processes mediated by the TGF $\beta$ pathway, especially those previously associated with $D K K 3$ overexpression, including invasion, angiogenesis, and chemoresistance. Although DKK3 has been described as a tumor suppressor in some cancers, $D K K 3$ is overexpressed in hepatocellular carcinoma and hepatoblastoma. ${ }^{6}$ The expression and function of DKK3, like the TGF $\beta$ pathway, depend on the tumor and tissue context. The overexpression and role of DKK3 have not been previously described in EAC.

In the current study, DKK3 was overexpressed in a significant subset of EACs. Esophageal cancers are heterogeneous, and most molecular changes, including DKK3 overexpression, will be present in only a subset of tumors. Although $D K K 3$ is overexpressed in $75.8 \%$ of EACs on RT-PCR, 2 to $3+$ DKK3 protein expression was found in $46.8 \%$ on immunohistochemistry. Discordance in mRNA and protein expression in other genes has been reported in lung adenocarcinoma. ${ }^{24}$ These differences may be the result of post-transcriptional regulation with changes in mRNA stability, ${ }^{25}$ transcript localization, ${ }^{26}$ or translational efficiency, as well as differences in sensitivity between mRNA and antibody-based assays.

The mechanism behind $D K K 3$ overexpression in EAC was also evaluated. $D K K 3$ was not amplified on SNP array analysis, and treatment of OE33 cells with 5-azacitidine alone did not increase DKK3 expression. However, a combination of 5-azacytidine and trichostatin A increased expression, suggesting histone acetylation is involved in $D K K 3$ overexpression.

The majority of patients with EAC die of metastatic disease. ${ }^{12}$ DKK3 overexpression may be important in tumor invasion, and Matrigel invasion was increased after transfection of DKK3 in Flo and OE33. Knockdown experiments could not be performed because none of the 3 available EAC cell lines overexpress DKK3. However, downstream inhibition of SMAD4 was able to decrease Matrigel invasion of OE33/DKK3. Deckers and colleagues $^{27}$ found that inhibiting SMAD4 in MDA-MB231 breast carcinoma cells inhibited bone metastases in nude mice. Although SMAD4 inhibition may have effects independent of $D K K 3$ overexpression, it is notable for its ability to inhibit Matrigel invasion of OE33/DKK3.

Neoangiogenesis is important in the progression from $\mathrm{BM}$ to adenocarcinoma and in supporting tumor invasion. ${ }^{28}$ DKK3 supports capillary formation in gliomas and lymphoma, ${ }^{8}$ and our results show an increase in endothelial tube formation in the presence of OE33/DKK3. Although transfection of DKK3 resulted in a more malignant phenotype with increased invasion and proliferation, there was no evidence that DKK3 is oncogenic in EAC. The 3 EAC cell lines available to us are cancer cell lines and therefore were not able to be specifically tested for oncogenic transformation. However, this chromosomal region was not amplified on SNP array.

To determine whether DKK3 transfection was able to increase the tumorigenicity of OE33, the lowest number of OE33/DKK3, but not OE33/Vector, cells able to produce tumors was determined to be $1 \times 10^{6}$ cells. This is several-fold less than previously described mice experiments using OE33 $\left(2 \times 10^{6}\right.$ to $1 \times 10^{7}$ cells $) .{ }^{29-32}$ In a tumorigenic assay, Zhao and colleagues ${ }^{18}$ injected 


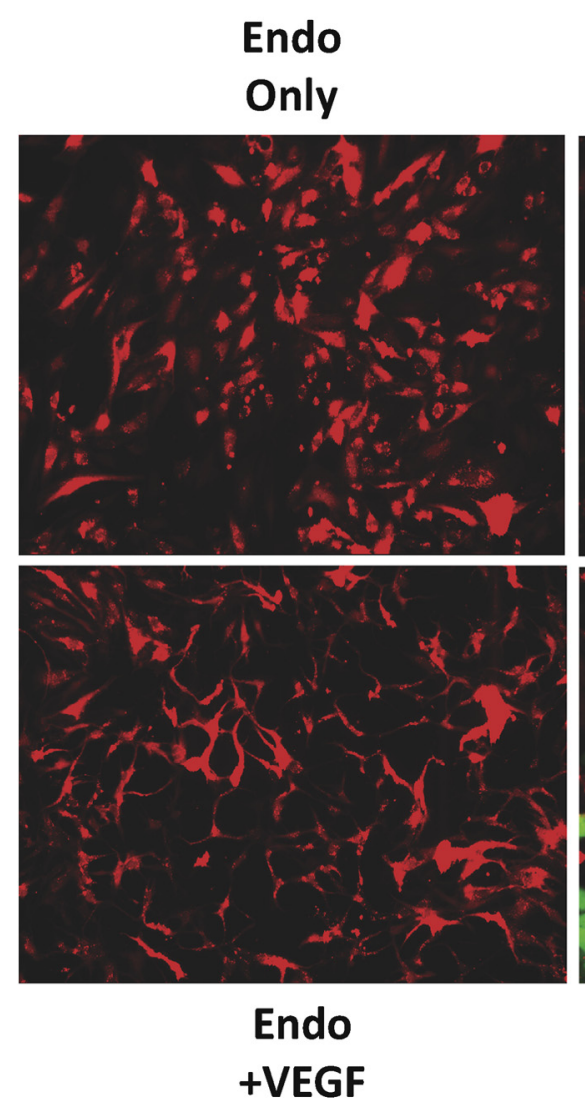

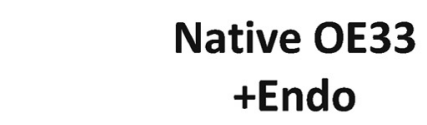
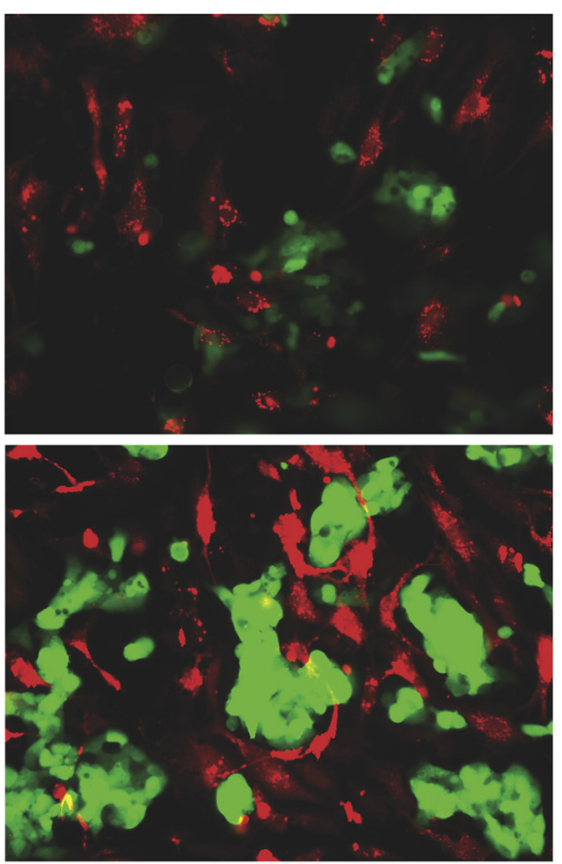

OE33/DKK3

+Endo
OE33/Vector

+Endo
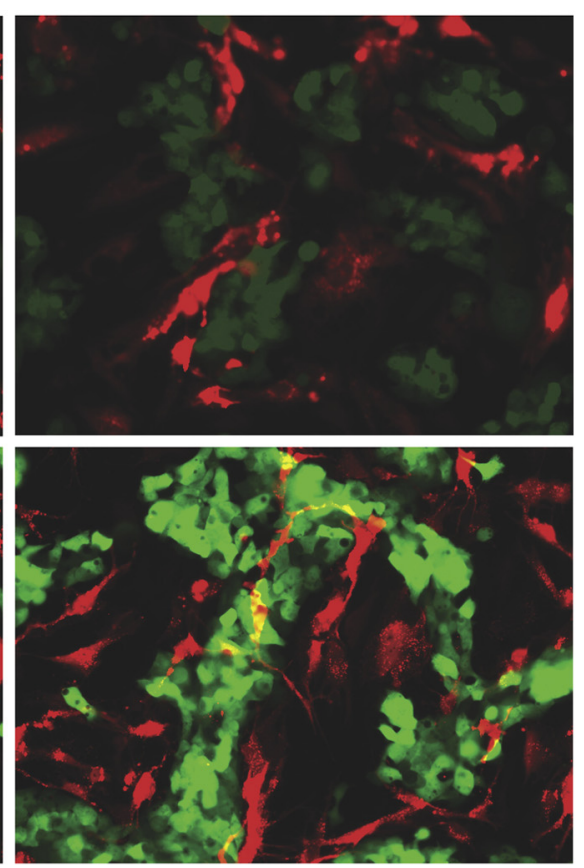

OE33/Vector +Endo+VEGF

FIGURE 4. The presence of stably transfected OE33 (OE33/DKK3 + Endo) increased endothelial tube formation similar to the tube formation seen after adding VEGF to endothelial cells (Endo + VEGF) and to OE33/Vector (OE33/Vector + Endo + VEGF). Endothelial, native OE33, and vector controls showed minimal tube formation. OE33 and endothelial cells were labeled green and red, respectively. Original magnifications were $200 \times$. VEGF, Vascular endothelial growth factor.

$1 \times 10^{5}, 1 \times 10^{6}$, and $1 \times 10^{7} \mathrm{OE} 33$ cells at 15 sites in BALB/c OlaHsd-Foxn $1^{\text {nu }}$ mice. OE33 cells produced tumors at 1 of 15 sites at 10 weeks and 2 of 15 sites at 29 weeks. In the current study, OE33/Vector cells produced tumors in 1 of 8 sites at 5 weeks, whereas OE33/DKK3 cells resulted in tumors at all 8 sites, supporting that $D K K 3$ expression is important in invasion and growth in EAC.

EACs highly overexpressing $D K K 3$ were significantly associated with nodal metastases in $88 \%(P=.047)$ and higher stage III or IVa in $81 \%$ of EACs $(P=.038)$, consistent with the increased Matrigel invasion seen with $D K K 3$ overexpression. Although $\mathrm{T}$ and $\mathrm{M}$ stage were not significantly associated with $D K K 3$ expression, this is likely because patients with invasion of surrounding structures or metastatic disease were not included in this series because they were not treated surgically.

Neoadjuvant chemoradiation followed by esophagectomy is standard in most large centers for EACs greater than T2 or with regional nodal disease to "downstage" the tumor and limit micrometastatic disease. However, only $21 \%$ of patients have a complete response. ${ }^{33} \mathrm{DKK} 3$ overexpression is associated with chemoresistance in Saos-2 osteosarcoma, ${ }^{9}$ and our results show that
OE33/DKK3 cells were significantly more chemoresistant to cisplatin and 5-FU. Targeting DKK3 and other TGF $\beta$ pathway mediators may decrease this chemoresistance. If inhibiting DKK3 alone is not sufficient, a combination with other therapies targeting the TGF $\beta$ pathway or downstream proteins may be a successful strategy.

DKK3-/- knockout mice have significantly decreased natural killer cells, have increased immunoglobulin-M and hemoglobin, and are hyperactive. ${ }^{34}$ Dkk3 does not appear to function as a tumor suppressor in these knockout mice because the authors did not report any increase in tumors. In addition, these knockout mice show that DKK3 is a nonlethal target because the $D K K 3-/-$ mice were viable.

\section{Study Limitations}

None of the 3 EAC cell lines overexpress $D K K 3$, so we were unable to evaluate targeted inhibition of $D K K 3$. However, we were able to evaluate the effect of transfection of $D K K 3$ in 2 EAC cell lines. Inhibition of $D K K 3$ using small interfering RNA in an oral squamous cell carcinoma cell line natively overexpressing $D K K 3$ significantly decreased migration and invasion, providing 

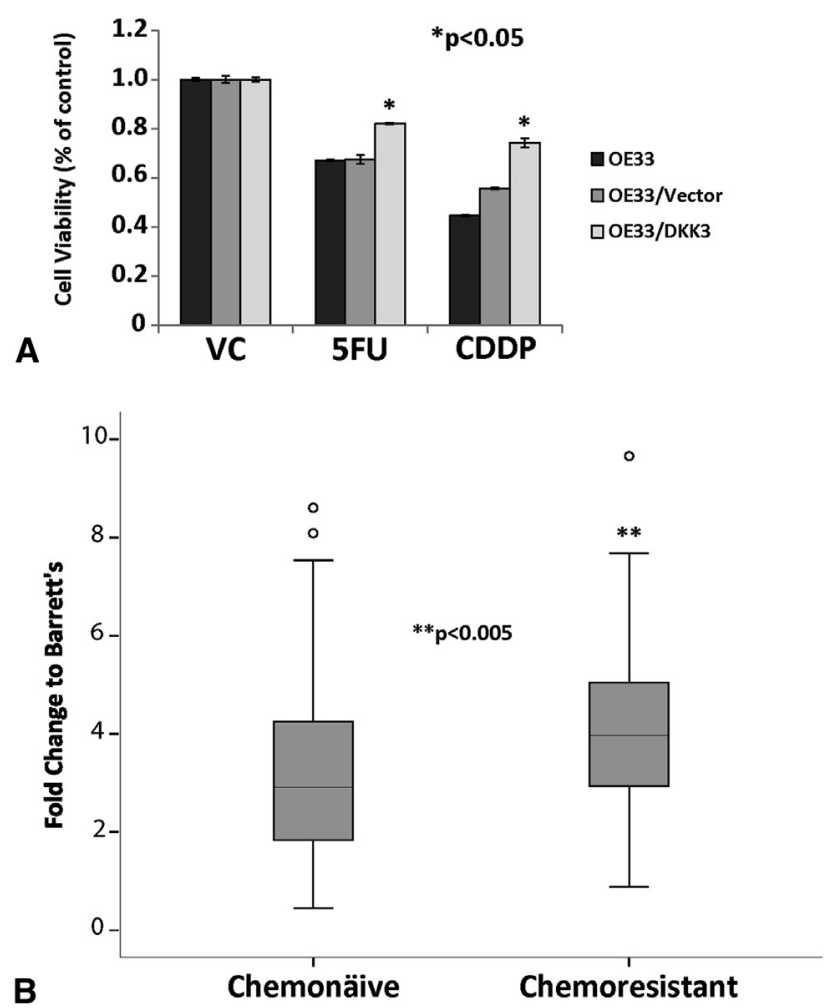

FIGURE 5. A, OE33/DKK3 were significantly more resistant to 5-FU and cisplatin compared with treatment with vehicle control for 48 hours $\left(*^{*}<.05\right)$. B, DKK3 expression was significantly higher on RT-PCR in chemoresistant $(\mathrm{n}=21)$ compared with chemonaive EACs $(\mathrm{n}=95)(* * P<.005) . V C$, Vector control; 5FU, 5-fluorouracil; $C D D P$, cis-diamminedichloroplatinum(II) (cisplatin); DKK3, Dickkopf-3.

further support that DKK3 plays a role in these biological processes. ${ }^{3 .}$

Although DKK3 overexpression was not associated with decreased overall survival, the sample size was relatively small and included only operable patients. $D K K 3$ overexpression was increased in chemoresistant tumors, and transfection increased chemoresistance and invasion. DKK3 overexpression may be associated with decreased survival in other populations, including patients with metastatic disease or those treated with preoperative chemotherapy. We were not able to evaluate these groups directly because our tumor bank consists mostly of chemonaive tumors obtained when patients were not treated routinely with preoperative chemotherapy. Katase and colleagues ${ }^{35}$ reported that patients with $D K K 3$ negative oral squamous cell carcinoma had decreased nodal metastases and significantly longer disease-free survival. These findings support that DKK3 overexpression is important clinically in cancers overexpressing DKK3.

\section{CONCLUSIONS}

The overall survival of patients with EAC remains poor, with the majority of patients ultimately dying of metastatic disease. The results of the current study suggest that DKK3 may play an important role in tumor growth and invasion in EAC. DKK3 is overexpressed in a significant subset of EACs, and targeting DKK3 and its downstream mediators may be beneficial in the prevention and treatment of micrometastatic disease and potentially decreasing disease recurrence.

\section{Conflict of Interest Statement}

Authors have nothing to disclose with regard to commercial support.

The authors thank David Erdody for help in preparing and formatting the images for this article.

\section{References}

1. Krupnik VE, Sharp JD, Jiang C, Robison K, Chickering TW, Amaravadi L, et al. Functional and structural diversity of the human Dickkopf gene family. Gene. 1999;238:301-13.

2. Mao B, Wu W, Li Y, Hoppe D, Stannek P, Glinka A, et al. LDL-receptor-related protein 6 is a receptor for Dickkopf proteins. Nature. 2001;411:321-5.

3. Nakamura RE, Hackam AS. Analysis of Dickkopf3 interactions with Wnt signaling receptors. Growth Factors. 2010;28:232-42.

4. Pinho S, Niehrs C. Dkk3 is required for TGF-beta signaling during Xenopus mesoderm induction. Differentiation. 2007;75:957-67.

5. Tsuji T, Miyazaki M, Sakaguchi M, Inoue Y, Namba M. A REIC gene shows down-regulation in human immortalized cells and human tumor-derived cell lines. Biochem Biophys Res Commun. 2000;268:20-4.

6. Pei Y, Kano J, Iijima T, Morishita Y, Inadome Y, Noguchi M. Overexpression of Dickkopf 3 in hepatoblastomas and hepatocellular carcinomas. Virchows Arch. 2009;454:639-46.

7. Zitt M, Untergasser G, Amberger A, Moser P, Stadlmann S, Zitt M, et al. Dickkopf-3 as a new potential marker for neoangiogenesis in colorectal cancer: expression in cancer tissue and adjacent non-cancerous tissue. Dis Markers. 2008;24:101-9.

8. Untergasser G, Steurer M, Zimmermann M, Hermann M, Kern J, Amberger A, et al. The Dickkopf-homolog 3 is expressed in tumor endothelial cells and supports capillary formation. Int J Cancer. 2008;122:1539-47.

9. Hoang BH, Kubo T, Healey JH, Yang R, Nathan SS, Kolb EA, et al. Dickkopf 3 inhibits invasion and motility of Saos-2 osteosarcoma cells by modulating the Wnt-beta-catenin pathway. Cancer Res. 2004;64:2734-9.

10. Hao Y, Triadafilopoulos G, Sahbaie P, Young HS, Omary MB, Lowe AW. Gene expression profiling reveals stromal genes expressed in common between Barrett's esophagus and adenocarcinoma. Gastroenterology. 2006;131:925-33.

11. Rhodes DR, Kalyana-Sundaram S, Mahavisno V, Varambally R, Yu J, Briggs BB, et al. Oncomine 3.0: genes, pathways, and networks in a collection of 18,000 cancer gene expression profiles. Neoplasia. 2007;9:166-80.

12. Siegel R, Naishadham D, Jemal A. Cancer statistics, 2012. CA Cancer J Clin. 2012;62:10-29.

13. Hsieh SY, Hsieh PS, Chiu CT, Chen W-Y. Dickkopf-3/REIC functions as a suppressor gene of tumor growth. Oncogene. 2004;23:9183-9.

14. Kononen J, Bubendorf L, Kallioniemi A, Bärlund M, Schraml P, Leighton S, et al. Tissue microarrays for high-throughput molecular profiling of tumor specimens. Nat Med. 1998;4:844-7.

15. Raso MG, Behrens C, Herynk MH, Liu S, Prudkin L, Ozburn NC, et al. Immunohistochemical expression of estrogen and progesterone receptors identifies a subset of NSCLCs and correlates with EGFR mutation. Clin Cancer Res. 2009;15:5359-68.

16. Bass AJ, Watanabe H, Mermel CH, Yu S, Perner S, Verhaak RG, et al. SOX2 is an amplified lineage-survival oncogene in lung and esophageal squamous cell carcinomas. Nat Genet. 2009;41:1238-42.

17. Wang B, Xiao Y, Ding BB, Zhang N, Yuan X-b, Gui L, et al. Induction of tumor angiogenesis by Slit-Robo signaling and inhibition of cancer growth by blocking Robo activity. Cancer Cell. 2003;4:19-29.

18. Zhao R, Quaroni L, Casson AG. Identification and characterization of stemlike cells in human esophageal adenocarcinoma and normal epithelial cell lines. J Thorac Cardiovasc Surg. 2012;144:1192-9. 
19. Helm J, Enkemann SA, Coppola D, Barthel JS, Kelley ST, Yeatman TJ. Dedifferentiation precedes invasion in the progression from Barrett's metaplasia to esophageal adenocarcinoma. Clin Cancer Res. 2005;11:2478-85.

20. Derynck R, Zhang YE. Smad-dependent and Smad-independent pathways in TGF-beta family signalling. Nature. 2003;425:577-84.

21. Derynck R, Akhurst RJ, Balmain A. TGF-beta signaling in tumor suppression and cancer progression. Nat Genet. 2001;29:117-29.

22. Kang YB. Pro-metastasis function of TGF beta mediated by the Smad pathway. J Cell Biochem. 2006;98:1380-90.

23. Seder CW, Hartojo W, Lin L, Silvers AL, Wang Z, Thomas DG, et al. Upregulated INHBA expression may promote cell proliferation and is associated with poor survival in lung adenocarcinoma. Neoplasia. 2009;11:388-96.

24. Chen G, Gharib TG, Huang CC, Taylor JM, Misek DE, Kardia SL, et al. Discordant protein and mRNA expression in lung adenocarcinomas. Mol Cell Proteomics. 2002;1:304-13.

25. Zhang Z, Sheng H, Shao J, Beauchamp RD, DuBois RN. Posttranscriptional regulation of cyclooxygenase-2 in rat intestinal epithelial cells. Neoplasia. 2000; $2: 523-30$

26. Lipshitz HD, Smibert CA. Mechanisms of RNA localization and translational regulation. Curr Opin Genet Dev. 2000;10:476-88.

27. Deckers M, van Dinther M, Buijs J, Que I, Lowik C, van der Pluijm G, et al. The tumor suppressor Smad4 is required for transforming growth factor beta-induced epithelial to mesenchymal transition and bone metastasis of breast cancer cells. Cancer Res. 2006;66:2202-9.

28. Sihvo EI, Ruohtula T, Auvinen MI, Koivistoinen A, Harjula AL, Salo JA. Simultaneous progression of oxidative stress and angiogenesis in malignant transformation of Barrett esophagus. J Thorac Cardiovasc Surg. 2003;126:1952-7.
29. Zhang YF, Zhang AR, Zhang BC, Rao ZG, Gao JF, Lv MH, et al. MiR-26a regulates cell cycle and anoikis of human esophageal adenocarcinoma cells through Rb1-E2F1 signaling pathway. Mol Biol Rep. 2013;40:1711-20.

30. Sehdev V, Katsha A, Ecsedy J, Zaika A, Belkhiri A, El-Rifai W. The combination of alisertib, an investigational Aurora kinase A inhibitor, and docetaxel promotes cell death and reduces tumor growth in preclinical cell models of upper gastrointestinal adenocarcinomas. Cancer. 2013;119:904-14.

31. Ford SJ, Obeidy P, Lovejoy DB, Bedford M, Nichols L, Chadwick C, et al Deferasirox (ICL670A) effectively inhibits oesophageal cancer growth in vitro and in vivo. Br J Pharmacol. 2013;168:1316-28.

32. Sehdev V, Peng D, Soutto M, Washington MK, Revetta F, Ecsedy J, et al. The aurora kinase A inhibitor MLN8237 enhances cisplatin-induced cell death in esophageal adenocarcinoma cells. Mol Cancer Ther. 2012;11:763-74.

33. Orringer MB, Marshall B, Chang AC, Lee J, Pickens A, Lau CL. Two thousand transhiatal esophagectomies: changing trends, lessons learned. Ann Surg. 2007; 246:363-74

34. Barrantes Idel B, Montero-Pedrazuela A, Guadano-Ferraz A, Obregon MJ, Martinez de Mena R, Gailus-Durner V, et al. Generation and characterization of dickkopf3 mutant mice. Mol Cell Biol. 2006;26:2317-26.

35. Katase N, Lefeuvre M, Gunduz M, Gunduz E, Beder LB, Grenman R, et al. Absence of Dickkopf (Dkk)-3 protein expression is correlated with longer disease-free survival and lower incidence of metastasis in head and neck squamous cell carcinoma. Oncol Lett. 2012;3:273-80.

Key Words: Esophageal adenocarcinoma, Dickkopf-3, DKK3, tissue invasion, chemoresistance

Readers who found these articles interesting may also like to read the following papers found in recent and future issues of our sister publications, Seminars in Thoracic and Cardiovascular Surgery and Operative Techniques in Thoracic and Cardiovascular Surgery!

\section{General Thoracic Surgery: Other Thoracic Surgical Papers}

Current Readings: David Zeltsman. Redefining Minimally Invasive Uniportal Video-Assisted Thoracic Surgery. Semin Thorac Cardiovasc Surg. Autumn 2014;26(3):249-254.

Current Readings: Sumeet Mittal. Failed Hiatus Hernia Repair. Semin Thorac Cardiovasc Surg. 2014;26(4):331-334.

State of the Art: Frank Detterbeck. The ITMIG Thymic Initiative: A State of the Art for the Study of Thymic Malignancies. Semin Thorac Cardiovasc Surg. 2014;26(4):317-322.

State of the Art: Richard Freeman. The Theory, Practice and Future of Process Improvement in General Thoracic Surgery. Semin Thorac Cardiovasc Surg. 2014;26(4):310-316.

State of the Art: Eric Grogan. Using Clinical Risk Models for Lung Nodule Classification. Semin Thorac Cardiovasc Surg. Expected Publication August 2015.

Original Submission: ThomasWatson. Eliminating a need for esophagectomy: endoscopic treatment of Barrett's Esophagus with early esophageal neoplasia. Semin Thorac Cardiovasc Surg. 2014;26(4):274-284.

Editorial Commentary: Anthony Kim. Endoscopically closing doors. Semin Thorac Cardiovasc Surg. 2014;26(4):285-286.

Original Submission: Daniela Molena. Incidence and risk factors for respiratory complications in patients undergoing esophagectomy for malignancy: a NSQIP analysis. Semin Thorac Cardiovasc Surg. 2014;26(4):287-294.

Editorial Commentary: R. Taylor Ripley. Esophageal complications: What are the real results? Semin Thorac Cardiovasc Surg. Expected publication April 2015. 2014;26(4):295-296.

Otis Rickman. Electromagnetic Navigation Assisted Bronchoscopy. Oper Tech Thorac Cardiovasc Surg. 2014;19(2):199-218.

Eric Grogan. Localization techniques for small lung nodules. Oper Tech Thorac Cardiovasc Surg. 2014;19(2):179-198.

Donald Nuss. The Minimally Invasive Repair of Pectus Excavatum. Oper Tech Thorac Cardiovasc Surg. 2014;19(3):324-347.

John Kucharczuk. Total Pectus Excavatum Repair: Open Approach. Oper Tech Thorac Cardiovasc Surg. 2014;19(3):348-364. 

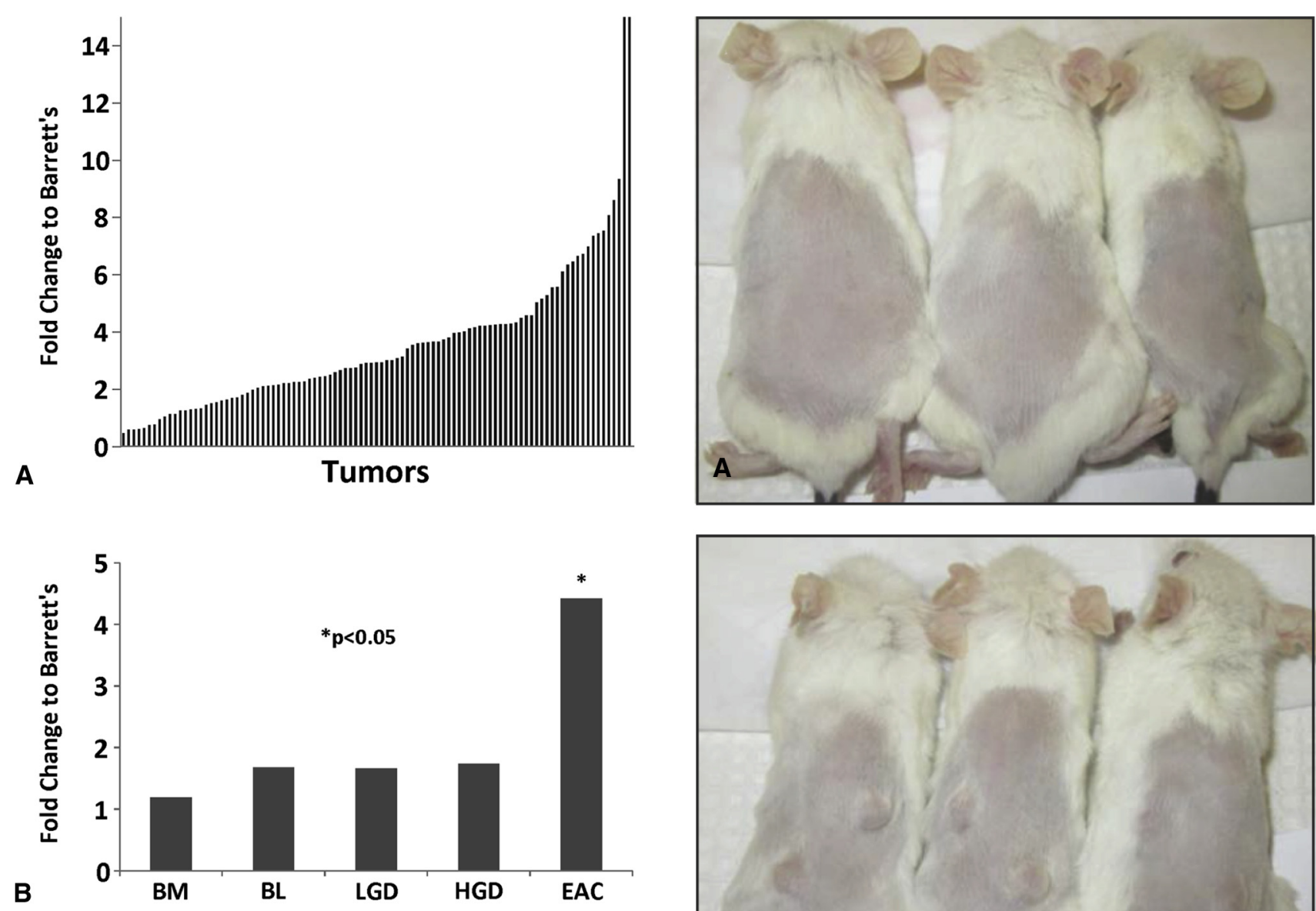

FIGURE E1. A, RT-PCR analysis of 95 EAC samples showed 2-fold overexpression of $D K K 3$ in $72 / 95(75.8 \%)$ EACs relative to BM. B, A total of 40 esophageal samples including 14 EACs showed significant overexpression in EAC in the progression from $\mathrm{BM}\left({ }^{*} P<.05\right)$. $B L$, Barrett's/low-grade dysplasia; $B M$, Barrett's metaplasia; $E A C$, esophageal adenocarcinoma; $L G D$, low-grade dysplasia; $H G D$, high-grade dysplasia.
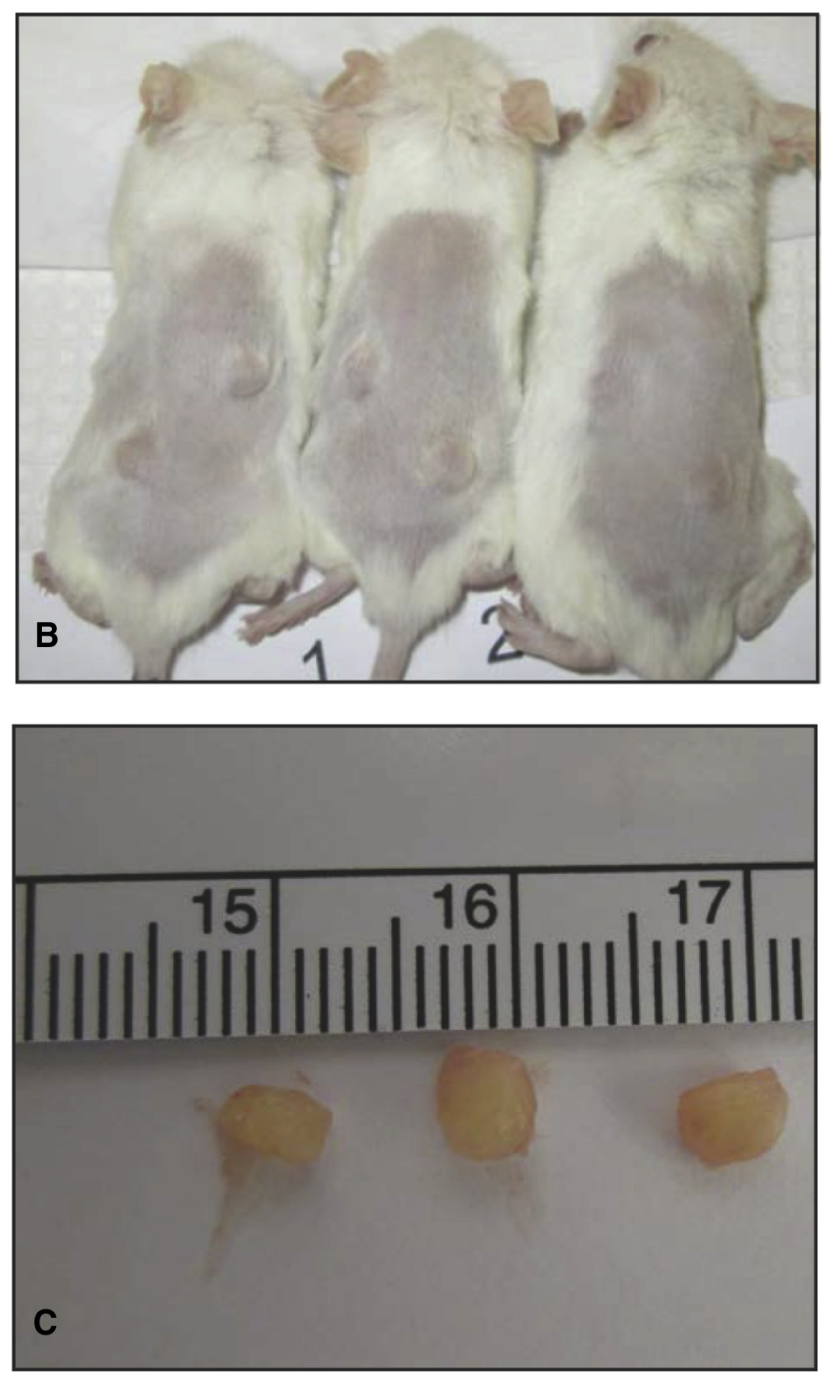

FIGURE E2. NOD/SCID $\gamma$ mice were injected in the flank with $1 \times 10^{6}$ cells. A, Only 1 of 8 OE33/Vector tumor sites produced a tumor $(2 \mathrm{~mm})$. $\mathrm{B}$, All (8/8) tumor sites injected with OE33/DKK3 cells resulted in tumors (4-5 mm) after 5 weeks (3/4 mice are shown). C, Three OE33/DKK3 tumors are shown after explant. DKK3 overexpression in the OE33/DKK3 tumors was confirmed on RT-PCR and Western blot. 
TABLE E1. Immunohistochemical analysis of Dickkopf-3 expression in esophageal tissues on tissue microarray*

\begin{tabular}{lcccc}
\hline & $\mathbf{0}$ & $\mathbf{1}+$ & $\mathbf{2}+$ & $\mathbf{3}+$ \\
\hline Normal esophagus & $0 / 2(0 \%)$ & $2 / 2(100 \%)$ & $0 / 2(0 \%)$ & $0 / 2(0 \%)$ \\
Barrett's metaplasia & $3 / 10(30 \%)$ & $5 / 10(50 \%)$ & $2 / 10(20 \%)$ & $0(0 \%)$ \\
Dysplasia & $1 / 12(8.3 \%)$ & $9 / 12(75 \%)$ & $2 / 12(16.7 \%)$ & $0(0 \%)$ \\
Adenocarcinoma & $11 / 62(17.7 \%)$ & $22 / 62(35.5 \%)$ & $24 / 62(38.7 \%)$ & $5 / 62(8.1 \%)$ \\
\hline
\end{tabular}

*Significant staining includes moderate to high staining (scores of 2 to $3+$ ).

TABLE E2. Clinical and pathologic characteristics of 94 chemonaive esophageal adenocarcinomas and DKK3 expression relative to Barrett's metaplasia on quantitative real-time polymerase chain reaction

\begin{tabular}{|c|c|c|c|c|}
\hline & $\begin{array}{c}D K K 3 \\
\text { Less than } \\
\text { mean }+2 \text { SD } \\
n=62\end{array}$ & $\begin{array}{c}D K K 3 \\
\text { Greater than } \\
\text { mean }+2 \text { SD } \\
\mathbf{n}=32\end{array}$ & $\begin{array}{c}\text { All } \\
\mathrm{n}=94\end{array}$ & $\begin{array}{c}P \\
\text { value }\end{array}$ \\
\hline Age, y, mean (SD) & $69.3(9.10)$ & $64(12.8)$ & $68.5(9.7)$ & .441 \\
\hline \multicolumn{5}{|l|}{ Gender } \\
\hline M & $55(89 \%)$ & $27(84 \%)$ & $82(87 \%)$ & \multirow[t]{2}{*}{.535} \\
\hline F & $7(11 \%)$ & $5(16 \%)$ & $12(13 \%)$ & \\
\hline \multicolumn{5}{|l|}{ Stage } \\
\hline $\mathrm{I}+\mathrm{II}$ & $26(42 \%)$ & $6(19 \%)$ & $32(34 \%)$ & \multirow[t]{2}{*}{.038} \\
\hline $\mathrm{III}+\mathrm{IVa}$ & $36(58 \%)$ & $26(81 \%)$ & $62(66 \%)$ & \\
\hline \multicolumn{5}{|l|}{ Barrett's } \\
\hline Y & $34(54 \%)$ & $15(47 \%)$ & $49(52 \%)$ & \multirow[t]{2}{*}{.524} \\
\hline $\mathrm{N}$ & $29(46 \%)$ & $17(51 \%)$ & $46(48 \%)$ & \\
\hline \multicolumn{5}{|l|}{ GEJ } \\
\hline $\mathrm{Y}$ & $23(37 \%)$ & $13(41 \%)$ & $36(38 \%)$ & \multirow[t]{2}{*}{.823} \\
\hline $\mathrm{N}$ & $40(63 \%)$ & $19(62 \%)$ & $59(62 \%)$ & \\
\hline \multicolumn{5}{|l|}{ T stage } \\
\hline 1 & $9(15 \%)$ & $1(3 \%)$ & $10(11 \%)$ & \multirow[t]{4}{*}{.132} \\
\hline 2 & $10(16 \%)$ & $3(9 \%)$ & $13(14 \%)$ & \\
\hline 3 & $41(66 \%)$ & $28(88 \%)$ & $69(73 \%)$ & \\
\hline 4 & $2(3 \%)$ & $0(0 \%)$ & $2(2 \%)$ & \\
\hline \multicolumn{5}{|l|}{$\mathrm{N}$ stage } \\
\hline 0 & $20(32 \%)$ & $4(12 \%)$ & $24(25 \%)$ & \multirow[t]{2}{*}{.047} \\
\hline 1 & $42(68 \%)$ & $28(88 \%)$ & $70(75 \%)$ & \\
\hline \multicolumn{5}{|l|}{ M stage* } \\
\hline 0 & $58(94 \%)$ & $26(81 \%)$ & $84(89 \%)$ & \multirow[t]{3}{*}{.084} \\
\hline 1a & $4(6 \%)$ & $6(19 \%)$ & $10(11 \%)$ & \\
\hline $1 \mathrm{~b}$ & $0(0 \%)$ & $0(0 \%)$ & $0(0 \%)$ & \\
\hline
\end{tabular}

$\overline{\text { Boldface indicates } P<.05 . D K K 3 \text {, Dickkopf-3; GEJ, gastroesophageal junction; }}$ $S D$, standard deviation. *M1a, positive celiac lymph nodes.TNM staging using AJCC Cancer Staging Manual. 6th ed. New York, NY: Springer; 2002. 